\title{
Optimal Investment and Risk Control Strategies for an Insurance Fund in Stochastic Framework
}

\author{
Patrick Kandege Mwanakatwe ${ }^{\circledR}$, Xiaoguang Wang, Yue Su \\ School of Mathematical Sciences, Dalian University of Technology, Dalian, China \\ Email: patrick26573@yahoo.co.uk, pkandege@mail.dlut.edu.cn
}

How to cite this paper: Mwanakatwe, P.K., Wang, X.G. and Su, Y. (2019) Optimal Investment and Risk Control Strategies for an Insurance Fund in Stochastic Framework. Journal of Mathematical Finance, 9, 254-265.

https://doi.org/10.4236/jmf.2019.93014

Received: April 30, 2019

Accepted: July 5, 2019

Published: July 8, 2019

Copyright () 2019 by author(s) and Scientific Research Publishing Inc. This work is licensed under the Creative Commons Attribution International License (CC BY 4.0).

http://creativecommons.org/licenses/by/4.0/

\begin{abstract}
This paper considers optimal investment and risk control problem under the Hull and White Stochastic Volatility (SV) model for an Insurer who aims to optimize the investment and risk control strategies. The surplus process of the insurer is assumed to follow the Brownian motion with drift. An Insurer can invest in the financial market consisting of risk-free and risky assets whose price process satisfies Hull-White SV model. By applying the stochastic dynamic programming approach, we derive closed-form expressions for the optimal strategies and the value function. We find that under the Hull and White model, the interest rate and risk aversion parameters both influence optimal strategies. Moreover, we provide a numerical example to illustrate the model's economic implications.
\end{abstract}

\section{Keywords}

Hull-White SV Model, Investment Policies, Stochastic Optimal Control, Reinsurance

\section{Introduction}

The insurance company is a financial intermediary obliged for compensation to a client if an uncertain event occurs. Its main goal is to protect the financial security of an individual, organization, or other entity in the case of unexpected loss through compensation. Since the discovery of first insurance risk model (Cramér-Lundberg model) at the beginning of the 20th century, many scholars have paid attention to study investment and risk control policies for Insurance Fund. For example, [1] [2] applied the classical Cramér-Lundberg model to describe the risk process where the insurer can invest in a risky asset only to minimize the ruin probability. Later, the studies [3] [4] [5] [6] [7] extended the model by considering investment in the risky asset, non-zero interest rate, and 
reinsurance to minimize the ruin probability. Moreover, [8] studied the optimal reinsurance and investment problem of minimizing the ruin probability. Also, [9] [10] considered the optimal asset allocation in the jump-diffusion process, where the compound Poisson risk process characterizes the claim process.

Furthermore, [11] [12] [13] introduced the Constant Elasticity of Variance (CEV) model into the optimal reinsurance and investment problem for insurers. In particular, [12] considered an optimal reinsurance-investment problem of an insurer whose surplus process follows a jump-diffusion model, [13] investigated the excess-of-loss reinsurance and investment problem for a compound Poisson jump-diffusion risk process. Likewise, [14] studied both an insurer's and a reinsurer's utilities.

Similarly, [11] [15] introduced zero-coupon bonds and Treasury Inflation-Protected Securities (TIPS) in the financial market. So the financial market consists of cash, zero-coupon bond, stock, and TIPS. While [15] assumed that the instantaneous nominal interest rate follows the Ornstein-Uhlenbeck process. They applied stochastic dynamic programming to derive the closed-forms of optimal reinsurance and investment strategies. [11] derived the time-consistent reinsurance-investment strategy under the mean-variance criterion for an insurer by applying the stochastic control theory.

The studies mentioned above, assume that the price processes of risky assets follow Geometric Brownian Motion, where the volatilities of risky assets are assumed to be constant or deterministic functions [16] [17]. This view is contrary to results found in the empirical results which support the presence of stochastic volatility (SV). In particular, [16] [17] applied Hestons SV model in the reinsurance. They obtained the optimal reinsurance and investment strategies with the stock price given by the Hestons SV model. For the meantime, SV is the current model which accommodates the volatility smile, the volatility clustering, and the heavy-tailed nature of return distributions. For more details about stochastic volatilities, a reader can refer to [18].

In this study, we assume that the price of risky assets follows the Hull and White Stochastic Volatility Model. The surplus process for the insurer follows Brownian motion with drift, and the insurer invests the surplus in risk-free and risky assets. Also, it is allowed to purchase proportional reinsurance as the risk control strategy. We first find the optimal investment and risk control strategies, by the first-order necessary condition for maximum. We also derive the optimal value function by plugging a reasonable conjecture into the HJB equation and solving nonlinear second-order partial differential equations. Moreover, we organize the rest of this paper as follows. In Section 2, we show various stages of model formulation, starting from classical risk model, financial market, by incorporating the Hull and White stochastic volatility model for the price of the risky asset and reinsurance policy. In Section 3, we present the value function with the corresponding HJB and derive the first order maximizing condition to our problem. In Section 4, we find an explicit solution for the problem by solving the HJB equation for CARA utility function. In Section 5, we demonstrate 
our model by a numerical example, and Section 6 concludes our work.

\section{Mathematical Model}

Assume that $\left(\Omega, \mathcal{F}_{t}, \mathcal{F}, \mathcal{P}\right)$ denotes a complete filtered probability space satisfying the general condition with a reference filtration $\mathcal{F}_{t \geq 0} . \mathcal{P}$ is a martingale probability measure equivalent to the real-world measure probability and $T>0$ is a time horizon.

\subsection{Risk Control Process}

Suppose that the surplus of the insurance company follows Brownian motion with drift. For a better understanding of the model formulation, we introduce the Cramér-Lundberg model as follows:

$$
R(t)=x_{0}+c t-Z(t), t>0
$$

$R(t)$ denotes the insurer's capital at time $t, x_{0}$ is the initial capital, $c>0$, $Z(t)$ denotes the premium income rate and the claims respectively. We also consider that the basis for calculating the premium rate of an insurer is the expected value principle. The respective mathematical expressions are:

$$
Z(t)=\sum_{i=1}^{N(t)} Y_{i}, i=1,2, \cdots, N(t) \text { and } c=(1+\theta) \lambda \mu .
$$

where $N(t)$ is the number of claims up to time $t$ and follows a Poisson process with intensity $\lambda>0$. Also $\theta>0$ is the safety loading interpreted as a risk premium rate and $Y_{i}$ denotes the ith claim and are independent and identically distributed random variables. To avoid the Insurer, from bankruptcy instantly we assume, $c>\lambda \mu$ as the necessary condition. Let the claim process $Z(t)$ follow the Brownian motion with drift:

$$
\mathrm{d} Z(t)=\mu \lambda \mathrm{d} t-\sigma \mathrm{d} W(t) .
$$

Therefore, the surplus process for insurance becomes:

$$
\mathrm{d} R(t)=c \mathrm{~d} t-\mathrm{d} Z(t)=\lambda \mu \theta \mathrm{d} t+\sigma \mathrm{d} W .
$$

Thus, the risk process is perturbed by Brownian motion and the insurer can choose proportional reinsurance over other types of reinsurance. Furthermore, we denote the risk exposure by $\alpha=[0,1]$, the proportional reinsurance level by $(1-\alpha)$ and the premium rate for the reinsurance is $c_{0}=(1+\phi) \lambda \mu$. Therefore, the insurer diverts a portion of the premium to the reinsurer at the rate of $(1+\phi) \lambda \mu(1-\alpha)$, where $\phi>0$ is the safety loading of the reinsurer and $\phi>\theta$. Thus, the surplus process $R(t)$ without investment satisfies the SDE:

$$
\mathrm{d} R(t)=\lambda \mu[\alpha(t)(1+\phi)-(\phi-\theta)] \mathrm{d} t+\alpha(t) \sigma \mathrm{d} W(t) .
$$

Following similar process as in [15] and references therein, and the expected value principle, the surplus diffusion process can be approximated by the following equation: 


$$
\mathrm{d} R(t)=\lambda \mu[(\theta-\phi)+\alpha(t) \phi] \mathrm{d} t+\alpha(t) \sigma \mathrm{d} W(t)
$$

\subsection{Financial Market}

Assume that the insurance company invests its surplus in the financial market where it is exposed to all of the common risk factors and hence prone to market fluctuations. Since the volatility is not constant, it is essential to model it as a risk factor of its own. The price process for a risk-free asset is given by:

$$
\mathrm{d} B(t)=B(t) r \mathrm{~d} t, B(0)=1, r>0,
$$

where $r>0$ is the free-risk interest rate. The dynamics of risky asset price is driven by SDE below:

$$
\begin{gathered}
\mathrm{d} S(t)=S(t)\left(\mu_{s} \mathrm{~d} t+\sqrt{v(t)} \mathrm{d} W_{s}(t)\right), S(0)>0, \\
\mathrm{~d} v(t)=k(\bar{v}-v(t)) \mathrm{d} t+w \mathrm{~d} W_{v}(t), v(0)>0,
\end{gathered}
$$

where $v(t)$ is the instant variance, $\mu_{s}$ is the appreciation rate, $k$ is the mean reversion, $\bar{v}$ is the long-run mean, and $w$ is the volatility-volatility (vol-vol) determining the variance of $v(t)$. Moreover, $W_{s}$ and $W_{v}$ are the standard Brownian motions with cross-variation satisfying the condition: $\mathrm{d}\left\langle W_{s}, W_{v}\right\rangle=\rho \mathrm{d} t$ with correlation $\rho \in[-1 ; 1]$. As noted in [18], the relationship between volatility and price is necessary to capture the so-called leverage effect, this effect is the tendency of volatility to increase as prices drop and decrease as prices rise.

\subsection{Wealth of an Insurance Fund}

We consider that the amount of wealth $X(t)$ invested in risky asset is $\beta(t)$ and the rest $(X(t)-\beta(t))$ is invested in riskless asset, and the reinsurance level is $\alpha(t)=[0,1]$. The dynamics of the wealth process of an Insurance Fund $X(t)$ is given by the SDE:

$$
\begin{aligned}
\mathrm{d} X(t)= & \lambda \mu[(\theta-\phi)+\alpha(t) \phi] \mathrm{d} t+\alpha(t) \sigma \mathrm{d} W(t) \\
& +\beta(t)\left(\mu_{s} \mathrm{~d} t+\sqrt{v(t)} \mathrm{d} W_{s}(t)\right)+(X(t)-\beta(t)) r, X(0)=x_{0}>0
\end{aligned}
$$

Proposition 2.1. The surplus process for an Insurance Fund evolve according to the $S D E$ :

$$
\begin{aligned}
\mathrm{d} X(t)= & {\left[\left(r X(t)+\beta(t)\left(\mu_{s}-r\right)\right)+\lambda \mu(\theta-\phi)\right] \mathrm{d} t } \\
& +\lambda \mu \alpha(t) \phi \mathrm{d} t+\sqrt{v(t)} \beta(t) \mathrm{d} W_{s}(t)+\alpha(t) \sigma \mathrm{d} W(t) .
\end{aligned}
$$

Definition 2.2. A strategy $\mathcal{A}=(\beta(t), \alpha(t))$ is said to be admissible if $\mathcal{A}$ is progressively measurable on the corresponding Brownian filtration $\mathcal{F}_{t}$ and satisfies the following conditions:
1) $\mathbf{E}\left(\int_{0}^{T} \alpha^{2}(t) \mathrm{d} t\right)<\infty$ a.s $\forall T<\infty$,
2) $\mathbf{E}\left(\int_{0}^{T} \beta^{2}(t) \mathrm{d} t\right)<\infty$ a.s $\forall T<\infty$. 
Therefore the SDE given by (9) has a strong unique solution, for all $\mathcal{A}=(\beta(t), \alpha(t))$.

Moreover, assume that the main objective of insurer is to maximize the expected utility of terminal wealth, the utility function $U(x)$ is continuous, twice differentiable and concave with $u^{\prime}>0$ and $u^{\prime \prime}<0$ respectively. Thus, the optimization problem is:

$$
\max _{\mathcal{A}}=\mathbf{E}[X(T)] \text { s.t. (7) and (9) }
$$

\section{The HJB Equation}

We derive the HJB equation by using the stochastic control method.

Proposition 3.1. Assume that $J(t, x, v) \in C^{(1,2,2)}$ such that the value function $J(t, v, x)$ and all its partial derivatives are continuous and differentiable, then the value function $J(t, v, x)$ satisfies the HJB equation:

$$
J_{t}+\sup _{(\beta(t), \alpha(t))} \Lambda\{J(t, x, v)\}=0, J(T, x, v)=U(x)
$$

$J(T, x, v)$ is the boundary condition, $\Lambda J(t, x, v)$ is:

$$
\begin{aligned}
& \max _{(\beta, \alpha)}\left\{\left[r x+\beta\left(\mu_{s}-r\right)+\lambda \mu(\alpha \phi-\phi+\theta)\right] J_{x}+k(\bar{v}-v) J_{v}\right. \\
& \left.+\frac{1}{2}\left(v \beta^{2}+\sigma^{2} \alpha^{2}\right) J_{x x}+\frac{1}{2}(w v)^{2} J_{v v}+w \beta \rho v \sqrt{v} J_{x v}\right\}=0 .
\end{aligned}
$$

Furthermore, we denote the partial derivatives for the value function by $J_{t}, J_{x}, J_{v}, J_{x x}, J_{v v}$ and $J_{x v}$.

Proposition 3.2. Assume that the HJB equation has a classical solution $J(t, v, x) \in C^{(1 ; 2 ; 2)}$, and fulfills the conditions that $J_{x}, J_{v}>0$, and $J_{x x}, J_{v v}, J_{x v}<0$. Then, maximum condition leads to:

$$
\beta^{*}(t, x, v)=-\frac{\left(\mu_{s}-r\right) J_{x}+w \rho v \sqrt{v} J_{x v}}{v J_{x x}},
$$

and

$$
\alpha^{*}(t, x)=-\frac{\lambda \mu \phi J_{x}}{\sigma^{2} J_{x x}} .
$$

where $\beta^{*}$, and $\alpha^{*}$ are the optimal investment and risk control strategies.

Then, substituting (12) and (13) into the HJB, we get the non-linear, second-order PDE:

$$
\begin{aligned}
& J_{t}+[r x+\lambda \mu(\theta-\phi)] J_{x}+k(\bar{v}-v) J_{v}+\frac{1}{2}(w v)^{2} J_{v v} \\
& -\frac{1}{2}(w v \rho)^{2} \frac{J_{x v}^{2}}{J_{x x}}-\frac{1}{2}\left[\frac{\left(\mu_{s}-r\right)^{2}}{v}+\left(\frac{\lambda \mu \phi}{\sigma}\right)^{2}\right] \frac{J_{x}^{2}}{J_{x x}} \\
& -\rho w\left(\mu_{s}-r\right) \sqrt{v} \frac{J_{x} J_{x v}}{J_{x x}}=0 .
\end{aligned}
$$




\section{The Optimal Strategies}

We solve the investment and risk control problem by maximizing the expected utility of terminal wealth. We choose the exponential utility (CARA) function for a risk averse insurer. This plays a prominent role in insurance practices because it is the only utility function under which the principle of zero utility gives a fair premium independent to the level of reserves of an insurance company. The CARA function is:

$$
U(x)=-\frac{1}{\gamma} \mathrm{e}^{-\gamma x}, \gamma>0 .
$$

We try to find the solution for (15) in the form:

$$
J(t, x, v)=-\frac{1}{\gamma} \exp \{-\gamma[a(t)(x-b(t))+g(t) v]\},
$$

substituting partial derivatives of (16) into (14) gives:

$$
\begin{aligned}
& {\left[a^{\prime}(t)(x-b(t))-a(t) b^{\prime}(t)+g^{\prime}(t) v\right]+k(\bar{v}-v) g(t)} \\
& +[r x+\lambda \mu(\theta-\phi)] a(t)-\frac{\gamma}{2}(w v)^{2} g^{2}(t)+\frac{\gamma}{2}(w v \rho)^{2} g^{2}(t) \\
& -(w \rho) v \sqrt{v}\left(\mu_{s}-r\right) g(t)+\frac{1}{2 \gamma}\left[\frac{\left(\mu_{s}-r\right)^{2}}{v}+\left(\frac{\lambda \mu \phi}{\sigma}\right)^{2}\right]=0 .
\end{aligned}
$$

Re-arranging (17) leads to:

$$
\begin{aligned}
& {\left[a^{\prime}(t)+r a(t)\right] x-a^{\prime}(t) b(t)-\left[b^{\prime}(t)-\lambda \mu(\theta-\phi)\right] a(t)} \\
& +v g^{\prime}(t)+k(\bar{v}-v) g(t)-w \rho v \sqrt{v}\left(\mu_{s}-r\right) g(t) \\
& -\frac{\gamma}{2}(w v)^{2}\left(1-\rho^{2}\right) g^{2}(t)+\frac{1}{2 \gamma}\left[\frac{\left(\mu_{s}-r\right)^{2}}{v}+\left(\frac{\lambda \mu \phi}{\sigma}\right)^{2}\right]=0 .
\end{aligned}
$$

Then, we split (18) as:

$$
\begin{gathered}
{\left[a^{\prime}(t)+r a(t)\right] x=0,} \\
a^{\prime}(t) b(t)-\left[b^{\prime}(t)-\lambda \mu(\theta-\phi)\right] a(t)+\frac{1}{2 \gamma}\left(\frac{\lambda \mu \phi}{\sigma}\right)^{2}=0 \\
v g^{\prime}(t)+\left[k(\bar{v}-v)-w \rho v \sqrt{v}\left(\mu_{s}-r\right)\right] g(t) \\
-\frac{\gamma}{2}(v w)^{2}\left(1-\rho^{2}\right) g^{2}(t)+\frac{1}{2 v \gamma}\left(\mu_{s}-r\right)^{2}=0
\end{gathered}
$$

Thus, we find the solutions for (19), (20) and (21) given boundary conditions $a(T)=1, b(T)=0$ and $g(T)=0$. For (19) we get:

$$
a(t)=\mathrm{e}^{r(T-t)} .
$$

Following simplifications of (20) we get the homogeneous first order DE:

$$
b^{\prime}(t)+r b(t)=\lambda \mu(\theta-\phi)+\frac{1}{2 \gamma}\left(\frac{\lambda \mu \phi}{\sigma}\right)^{2} \mathrm{e}^{-r(T-t)}
$$

By considering boundary condition $b(T)=0$ we get: 


$$
b(t)=\frac{1}{r}\left(\mathrm{e}^{r(T-t)}-1\right)\left[\lambda \mu(\theta-\phi)+\frac{1}{2 \gamma}\left(\frac{\lambda \mu \phi}{\sigma}\right)^{2} \mathrm{e}^{-r(T-t)}\right]
$$

Again, re-writing Equation (21) simplifies to:

$$
\begin{aligned}
& g^{\prime}(t)-\frac{\gamma}{2 v}(v w)^{2}\left(1-\rho^{2}\right) g^{2}(t)+\frac{1}{v}\left[k(\bar{v}-v)-w \rho v \sqrt{v}\left(\mu_{s}-r\right)\right] g(t) \\
& +\frac{1}{2 \gamma}\left(\frac{\mu_{s}-r}{v}\right)^{2}=0 .
\end{aligned}
$$

Equation (25) is a Riccati DE. For more simplification purposes, we denote $A$, $B$ and $C$ respectively by:

$$
\left\{\begin{array}{l}
A=-\frac{1}{2} \gamma v w^{2}\left(1-\rho^{2}\right) \\
B=\frac{k(\bar{v}-v)-w \rho v \sqrt{v}\left(\mu_{s}-r\right)}{v} \\
C=\frac{1}{2 \gamma v^{2}}\left(\mu_{s}-r\right)^{2}
\end{array}\right.
$$

Then for the notational convenience Equation (25) turns to:

$$
g^{\prime}(t)+A g^{2}(t)+B g(t)+C=0
$$

Consideration the boundary condition, $g(T)=0$, the direct calculations for the Riccati Differential Equation leads to:

$$
g(t)= \begin{cases}y_{1}-\frac{2 y_{1} A+B}{A\left(\mathrm{e}^{-\left(2 y_{1} A+B\right)(-t+T)}-1\right)}, & \text { for } \rho \neq \pm 1 \\ \frac{A\left(\mathrm{e}^{B(-t+T)}-1\right)}{B}, & \text { for } \rho= \pm 1\end{cases}
$$

Hence, we can summarise the above by the theorem.

Theorem 4.1. With reference to the value function given by Equation (11), the optimal strategies for an insurance company are defined as.

$$
\begin{gathered}
\beta^{*}(t)=\frac{\left(\mu_{s}-r\right)-\gamma w \rho v \sqrt{v} g(t)}{\gamma v a(t)}, \\
\alpha^{*}(t)=\frac{\mu \lambda \phi}{\gamma \sigma^{2} a(t)}, \\
J(t, x, v)=-\frac{1}{\gamma} \exp \{-\gamma[a(t)(x-b(t))+g(t) v]\} .
\end{gathered}
$$

where $\beta^{*}(t)$ and $\alpha^{*}(t)$ are the optimal investment, and risk control strategies, $J(t, x, v)$ is the value function. Moreover, $a(t), g(t)$, and $b(t)$ are given by Equations (22), (24), and (28) respectively.

\section{Numerical Illustration}

This section, presents some numerical simulations to illustrate the model. The parameters used in simulation are given as; Financial Market Parameters: 
$\mu_{s}=0.09, \bar{v}=0.04, k=0.02, w=0.08, \rho=0.30, r=0.05$, and Insurance Parameters: $\theta=0.2, \phi=0.4, \sigma=1.0, \mu \lambda=4.0, T=10, \gamma=1.2$.

\subsection{Analysis of Interest Rate and Risk Aversion on Optimal Strategies}

Figure 1 and Figure 2 show the effect of the interest rate on optimal investment, and reinsurance strategies respectively. Figure 1 and Figure 2 shows that the larger interest rate $r$, the optimal reinsurance and investment strategies tend to decrease. The key reason for this is that with the increase of $r$, the risk-free asset becomes more attractive. Hence the insurer is more likely to invest more in the risk-free asset instead of purchasing more reinsurance.

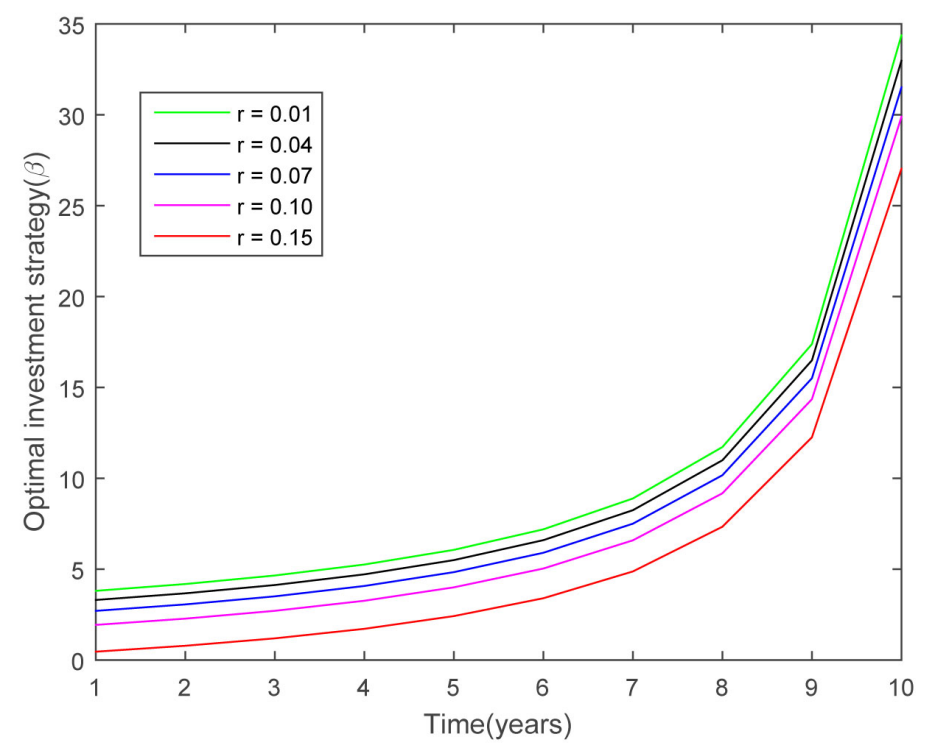

Figure 1. Influence of $r$ on $\beta$.

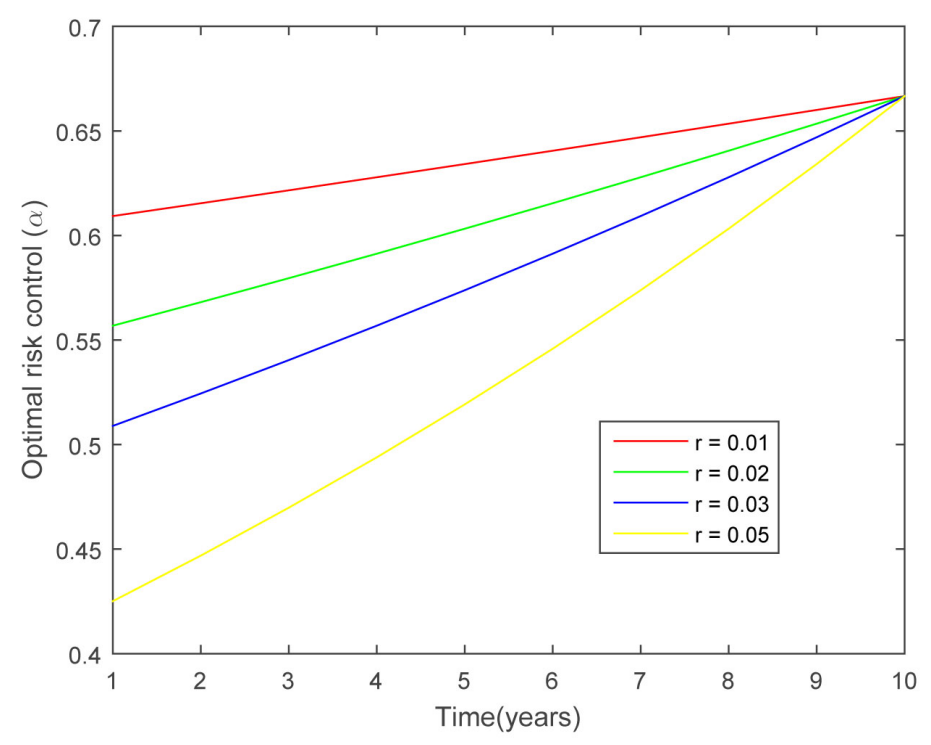

Figure 2. Influence of $r$ on $\alpha$. 
Figure 3 and Figure 4 present the fluctuations in the optimal strategies under different risk aversion levels. In particular, we find that $\gamma$ exerts an adverse effect on $\beta$ and $\alpha$. Figure 3 shows that the larger the degree of risk aversion $\gamma$, the more the risk averse the investor. Also, we have an explicit knowledge that the more risk averse investor, will invest less amount of the wealth in the risky asset to avoid risk. Figure 4 shows that the optimal reinsurance strategy increases as the risk aversion $\gamma$ decreases, this implies that the larger coefficient of risk aversion the more risk averse the insurer. Therefore, the insurer can buy more reinsurance to spread risk.

\subsection{Analysis of the Optimal Investment Strategy}

The effect brought by the volatility of volatility $w$ on the investment strategy is

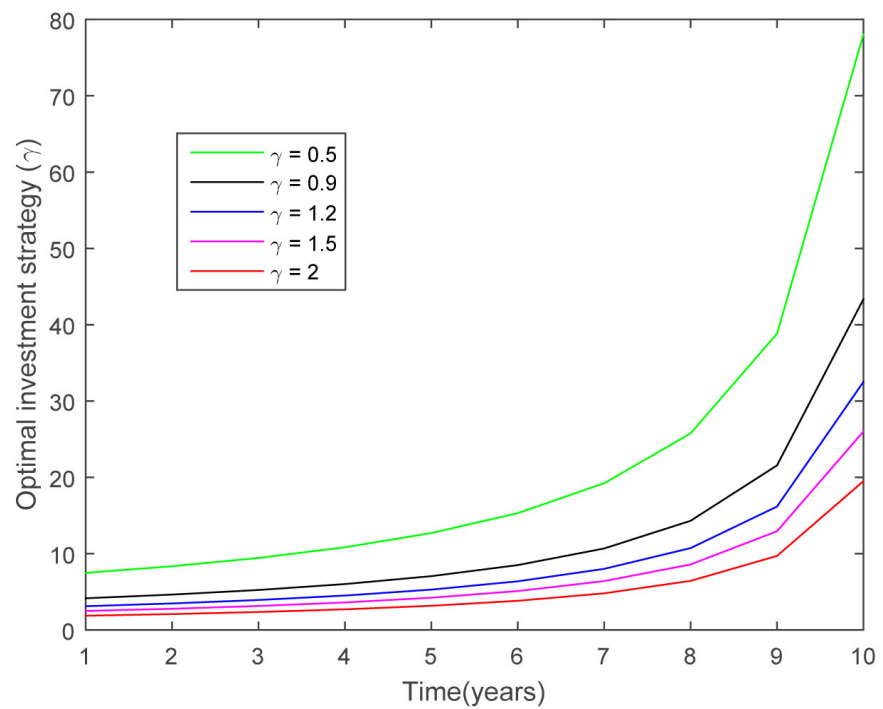

Figure 3. Influence of $\gamma$ on $\beta$.

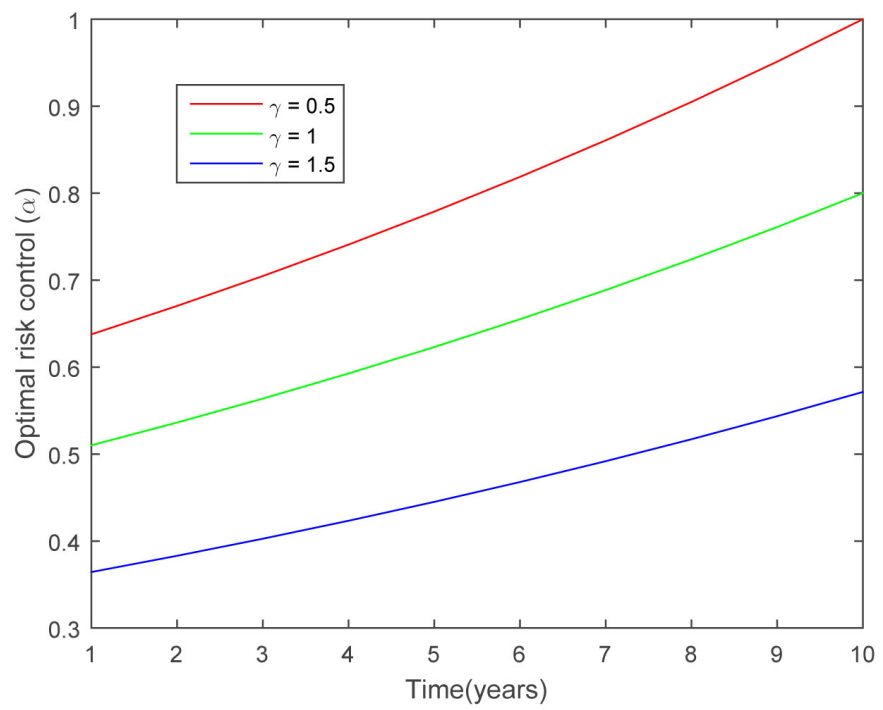

Figure 4. Influence of $\gamma$ on $\alpha$. 
presented in Figure 5. It shows that, if $\rho>0$, the optimal investment strategy increases with decrease in volatility of volatility $w$. Thus if $w$ declines, the volatility of the risky asset fluctuates drastically. Thus the insurer has to reduce investment in the risky asset as $w$ decreases. Figure 6 shows similar features on the effect of volatility $v$ on the optimal investment strategy. Furthermore, it shows that the optimal investment policy decreases if $\rho<0$ and increases if $\rho>0$. That is, if $\rho>0$, the risky asset price and its volatility process move in the same direction.

\subsection{Analysis of the Optimal Reinsurance}

Figure 7 shows that the optimal reinsurance strategy $\alpha(t)$ increases with the decrease in the volatility of the surplus process $\sigma$, that is, if the risk of the insurance business decreases, the insurer have to buy less reinsurance or acquire more new business.

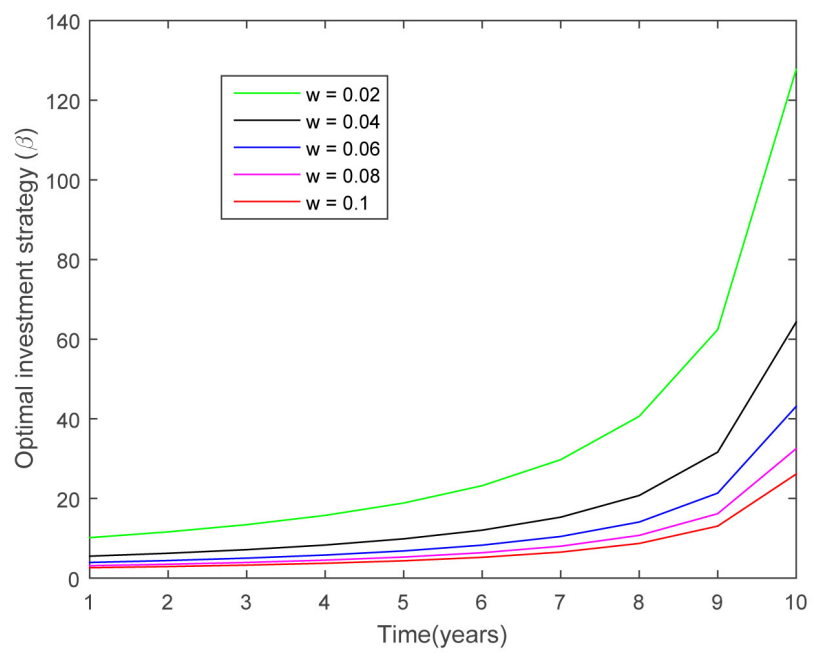

Figure 5. Impact $w$ on $\beta$.

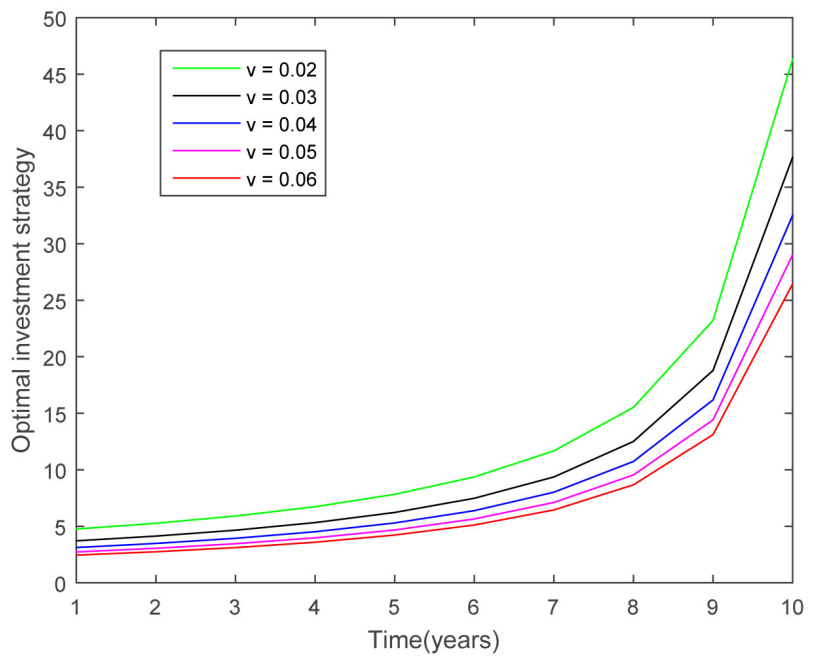

Figure 6. Effect of $v$ on $\beta$. 


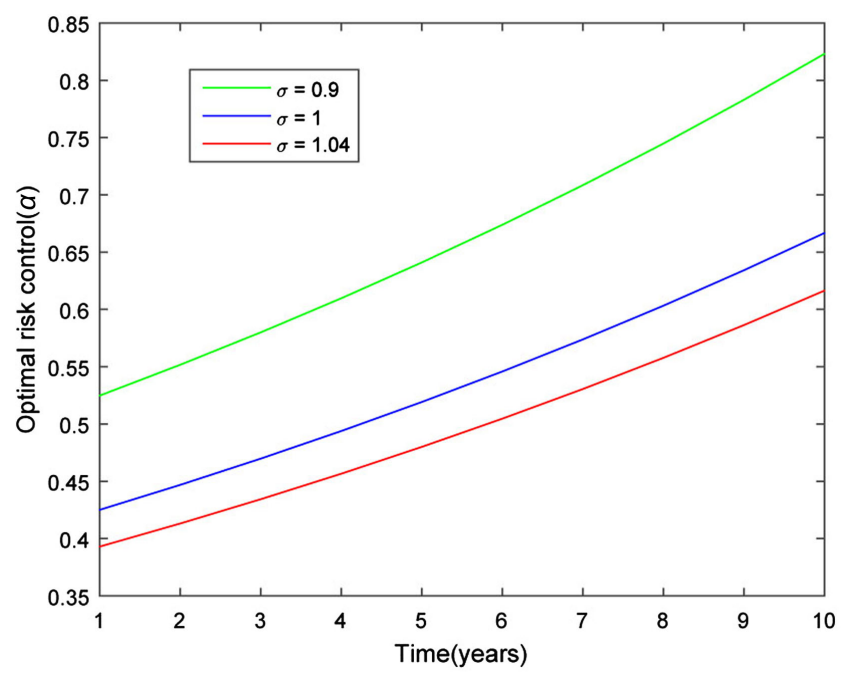

Figure 7. Effect of $\sigma$ on $\alpha$.

\section{Conclusion}

We considered that the surplus process for the insurer follows Brownian motion with drift. The insurer is allowed to buy proportional reinsurance and can invest the surplus in a financial market comprising of risk-free and risky assets. We assume that the volatility of a risky asset follows the Hull and White SV model. We look for the optimal investment-reinsurance strategy to maximize the expected exponential utility of terminal wealth. Using stochastic control approach and the HJB equation, we obtain exact solutions for the optimal strategies and derive the value function.

\section{Conflicts of Interest}

The authors declare no conflicts of interest regarding the publication of this paper.

\section{References}

[1] Embrechts, P., Klüppelberg, C. and Mikosch, T. (2013) Modelling Extremal Events: For Insurance and Finance. Vol. 33, Springer Science \& Business Media, Berlin.

[2] Hipp, C. and Plum, M. (2000) Optimal Investment for Insurers. Insurance: Mathematics and Economics, 27, 215-228. https://doi.org/10.1016/S0167-6687(00)00049-4

[3] Liu, C.S. and Yang, H. (2004) Optimal Investment for an Insurer to Minimize Its Probability of Ruin. North American Actuarial Journal, 8, 11-31. https://doi.org/10.1080/10920277.2004.10596134

[4] Schmidli, H. (2002) On Minimizing the Ruin Probability by Investment and Reinsurance. Annals of Applied Probability, 12, 890-907. https://doi.org/10.1214/aoap/1031863173

[5] Taksar, M.I. and Markussen, C. (2003) Optimal Dynamic Reinsurance Policies for Large Insurance Portfolios. Finance and Stochastics, 7, 97-121. https://doi.org/10.1007/s007800200073

[6] Wang, Z., Xia, J. and Zhang, L. (2007) Optimal Investment for an Insurer: The Mar- 
tingale Approach. Insurance: Mathematics and Economics, 40, 322-334. https://doi.org/10.1016/j.insmatheco.2006.05.003

[7] Yang, H. and Zhang, L. (2005) Optimal Investment for Insurer with Jump-Diffusion Risk Process. Insurance: Mathematics and Economics, 37, 615-634. https://doi.org/10.1016/j.insmatheco.2005.06.009

[8] Luo, S., Taksar, M. and Tsoi, A. (2008) On Reinsurance and Investment for Large Insurance Portfolios. Insurance: Mathematics and Economics, 42, 434-444. https://doi.org/10.1016/j.insmatheco.2007.04.002

[9] Lin, C., Jin, P., Bo, Z. and Rosyida, I. (2017) Diversified Models for Portfolio Selection Based on Uncertain Semi Variance. International Journal of Systems Science, 48, 637-648. https://doi.org/10.1080/00207721.2016.1206985

[10] Laubis, B. and Lin, J.E. (2008) Optimal Investment Allocation in a Jump Diffusion Risk Model with Investment: A Numerical Analysis of Several Examples. Proceedings of the 43rd Actuarial Research Conference, 14-16 August 2008, 1-21.

[11] Li, D., Rong, X. and Zhao, H. (2015) Time-Consistent Reinsurance-Investment Strategy for an Insurer and a Reinsurer with Mean-Variance Criterion under the CEV Model. Journal of Computational and Applied Mathematics, 283, 142-162. https://doi.org/10.1016/j.cam.2015.01.038

[12] Lin, X. and Li, Y. (2011) Optimal Reinsurance and Investment for a Jump Diffusion Risk Process under the CEV Model. North American Actuarial Journal, 15, 417-431. https://doi.org/10.1080/10920277.2011.10597628

[13] Sheng, D.L., Rong, X. and Zhao, H. (2014) Optimal Control of Investment-Reinsurance Problem for an Insurer with Jump-Diffusion Risk Process: Independence of Brownian Motions. Abstract and Applied Analysis, 2014, Article ID: 194962. https://doi.org/10.1155/2014/194962

[14] Li, D., Rong, X. and Zhao, H. (2014) Optimal Reinsurance-Investment Problem for Maximizing the Product of the Insurer's and the Reinsurer's Utilities under a CEV Model. Journal of Computational and Applied Mathematics, 255, 671-683. https://doi.org/10.1016/j.cam.2013.06.033

[15] Guan, G. and Liang, Z. (2014) Optimal Reinsurance and Investment Strategies for Insurer under Interest Rate and Inflation Risks. Insurance: Mathematics and Economics, 55, 105-115. https://doi.org/10.1016/j.insmatheco.2014.01.007

[16] Li, Z., Zeng, Y. and Lai, Y. (2012) Optimal Time-Consistent Investment and Reinsurance Strategies for Insurers under Heston's SV Model. Insurance: Mathematics and Economics, 51, 191-203. https://doi.org/10.1016/j.insmatheco.2011.09.002

[17] Zhao, H., Rong, X. and Zhao, Y. (2013) Optimal Excess-of-Loss Reinsurance and Investment Problem for an Insurer with Jump-Diffusion Risk Process under the Heston Model. Insurance: Mathematics and Economics, 53, 504-514. https://doi.org/10.1016/j.insmatheco.2013.08.004

[18] Musiela, M. and Rutkowski, M. (2006) Martingale Methods in Financial Modelling. Vol. 36, Springer Science \& Business Media, Berlin. https://doi.org/10.1007/b137866 